\title{
Towards the Automated Coverlay Assembly in FPCB Manufacturing: Concept and Preliminary Tests
}

\author{
Marcello Valori ${ }^{1(\bowtie)}\left(\mathbb{D}\right.$, Vito Basile ${ }^{1}\left(\mathbb{0}\right.$, Simone Pio Negri $^{1}\left(\mathbb{D}\right.$, Paolo Scalmati ${ }^{2}$, \\ Chiara Renghini ${ }^{2}$, and Irene Fassi ${ }^{3}$ \\ ${ }^{1}$ Institute of Intelligent Industrial Technologies and Systems for Advanced Manufacturing, \\ National Research Council, Via P. Lembo, 38/F, 70124 Bari, Italy \\ marcello.valoriastiima.cnr.it \\ 2 Somacis S.p.A, Via del Lauro, 7, 20121 Milan, Italy \\ \{p.scalmati, c.renghini\} @somacis.com
}

3 Institute of Intelligent Industrial Technologies and Systems for Advanced Manufacturing, National Research Council, Via A. Corti, 12, 20133 Milan, Italy

\begin{abstract}
In modern electronics, flexible and rigid-flex PCBs are largely used due to their intrinsic versatility and performance, allowing to increase the available volume, or enabling connection between unconstrained components. Rigid-flex PCBs consists of rigid board portions with flexible interconnections and are commonly used in a wide variety of industrial applications. However, the assembly process of these devices still has some bottlenecks. Specifically, they require the application of cover layers (namely, coverlays), to provide insulation and protection of the flexible circuits. Due to the variability in planar shape and dimensions, the coverlay application is still performed manually, requiring troublesome manipulation steps and resulting in undetermined time-cycle and precision.

This paper aims at the improvement of the industrial process currently performed, by proposing an approach for the automation of Kapton coverlay manipulation and application. Since these products are commercially provided as a film with a protective layer to be removed, the peeling issue is addressed, representing a challenging step of the automated process; the results of a systematic series of tests, performed in order to validate the peeling strategy, are reported in the paper. The overall assembly strategy relies on the development of a customized multi-hole vacuum gripper, whose concept is presented and contextualized in the proposed assembly process by outlining a suitable workcell architecture.
\end{abstract}

Keywords: PCB assembly $\cdot$ Film manipulation $\cdot$ Flexible electronics

\section{Introduction}

Modern electronics is moving fast, chasing market demands, towards miniaturization, flexible and stretchable electronics [1], embedded PCBs [2], molded interconnect devices (MIDs) and 3D electronic devices [3], environment friendly electronics [4]. Among these 
trends, flexible and rigid-flex PCBs are used in many applications due to their advantages compared to traditional rigid PCBs: by interposing flexible connections between rigid board portions, complex 3D arrangements can be achieved, complying with demanding volume constraints.

The main advantage obtained by the use of flexible printed circuit boards (FPCBs) is the possibility of 3D wiring, enabling the exploitation of the available volumes and complex routing requirements. In particular, exploiting their intrinsic lightweight and the possibility to be deformed and constrained into narrow volumes, flexible circuits are commonly used in biomedical applications [5], aerospace and smartphone industry. One fundamental feature of flexible circuits is the necessity of mechanical protection of the conductors; in order to comply with this requirement, coverlays are applied in addition to the solder masks. Among the various types of coverlay (film, screen printable, photoimageable) [6], film coverlay is generally preferred, due to its mechanical properties in terms of strength and durability. However, it is the most complicated solution from the point of view of the automation possibilities. Film coverlay thickness can span from $25 \mu \mathrm{m}$ to $127 \mu \mathrm{m}$; the base material, polyimide (PI) or Polyethylene terephthalate (PET), is coated with an adhesive layer of epoxy resin or acrylic with thicknesses in the range $25 \div 75 \mu \mathrm{m}$. The adhesives are semicured, to be activated finally in the lamination phase. A further layer of release material is applied as adhesive layer protection for delivery and handling before application.

Figure 1 shows an example of rigid-flexible PCB layer stack-up. Figure 2 shows two examples of rigid-flexible circuits developed by the PCB manufacturer Somacis, Italy, as well as the effects of a final "routing" phase, referred in this case to the full-thickness trimming of the external frame to actualize the flexibility. Light brown, flexible circuits can be distinguished from green rigid circuits; the different color is due to coverlay surface. In some cases, flexible circuits enable signal connections between rigid circuits (Fig. 2, a), while in other cases they embody sensors or other devices (Fig. 2, b).

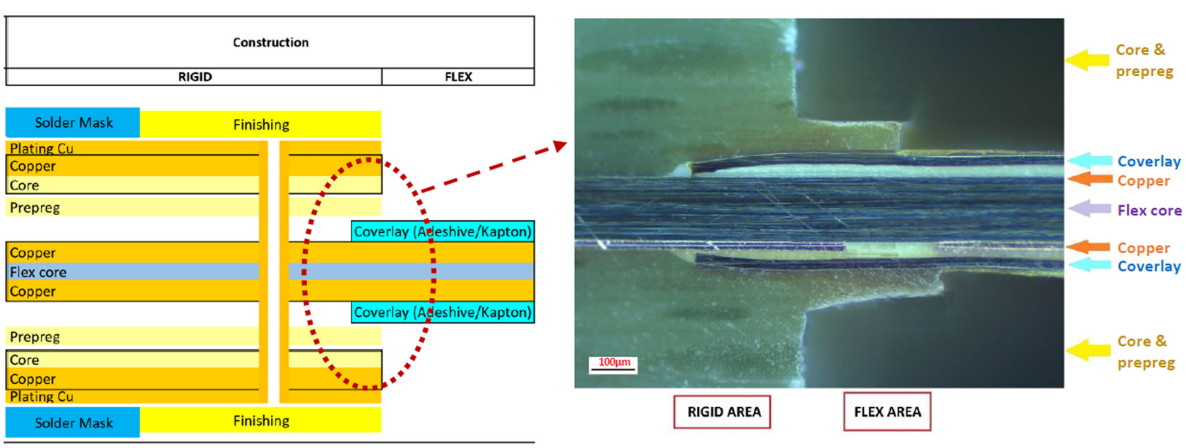

Fig. 1. Rigid-flexible PCB stack-up. Left: schematic of the layers. Right: a detail of the assembled circuit section acquired by a Zeiss Axio Imager M2m microscope. (Courtesy of Somacis, Italy).

In Fig. 3, a flexible circuit portion is shown, before and after the application of the coverlay; as highlighted, coverlay application is limited to flexible areas of the circuit, specific for each board design. The actual flexibility is obtained when, furtherly, the 

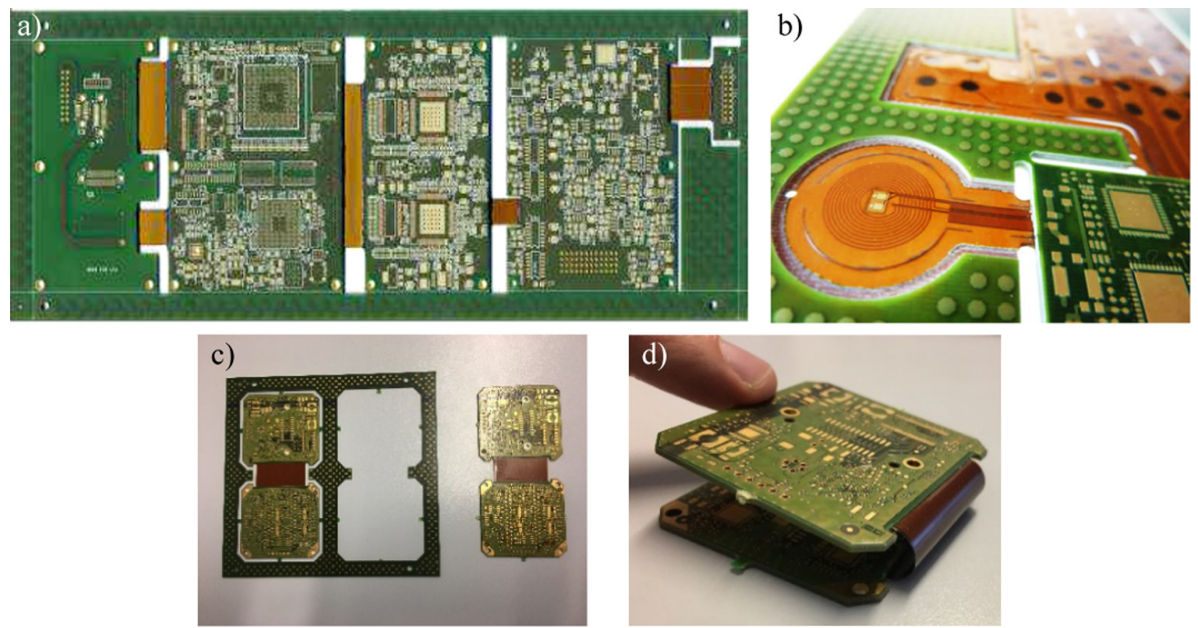

Fig. 2. Rigid-flexible circuits. a) Industrial application board, 14 layers (10 rigid +4 flexible), thickness $2 \mathrm{~mm}$. b) Device for human epilepsy monitoring. c) and d) The external frame furtherly eliminated, enabling the deformation of flexible circuits. (Courtesy of Somacis, Italy).

external frame, that is necessary for automated manipulation during the assembly of the other components, is removed. It is worth to highlight that the removal of the coverlay protective film can be troublesome, increasing process time, thus production costs, and inducing visual and mental stress for the worker.
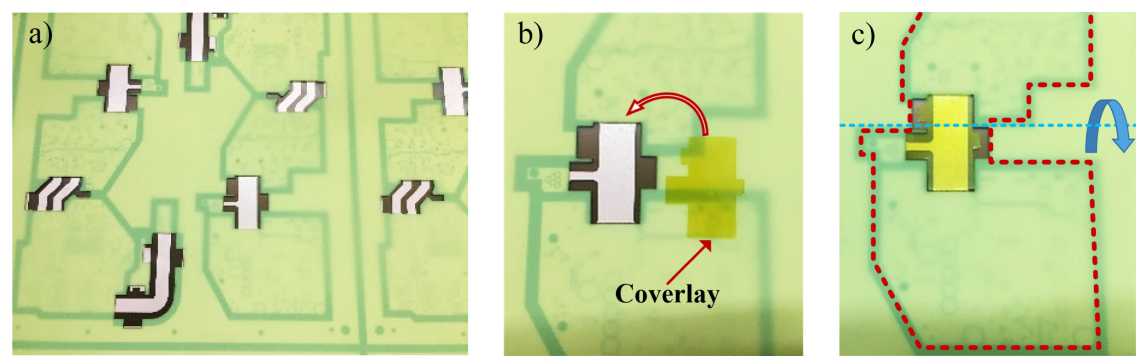

Fig. 3. Coverlay application. Flexible circuit before (a) and after (c) the application of the coverlay (b). In c) the red countour highlights the frame to be trimmed out and the blue line indicates the flexion axis of the final device. (Courtesy of Somacis, Italy). (Color figure online)

Flexible circuits layers are laminated together with rigid layers, according to the designed build-up (Fig. 4, left). In PCB fabrication, indeed, the layer stack-up is preassembled and then subjected to lamination and curing (pressure-heating cycle), aimed at the activation of the adhesive on coverlays and flexible circuits and the polymerization of epoxy resin in prepreg foils (Fig. 4, right).

The state-of-the-art application process of film coverlays is characterized by the following steps (Fig. 5): a) laser trimming of the specific contour, b) manual removal of the protective film, c) manual positioning on the PCB, d) manual application of one 

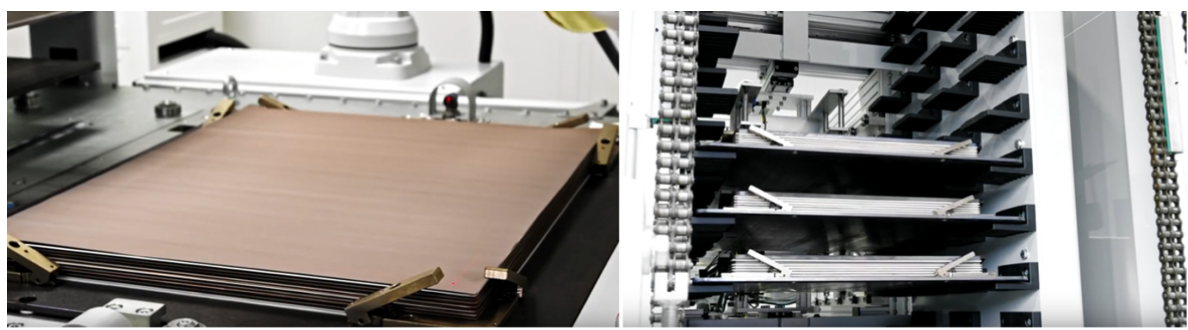

Fig. 4. PCB fabrication: lamination and curing cycle. Pre-assembled stack-up (left) and 20 positions loader of a Lauffer press. (Courtesy of Somacis, Italy)

or more welding points to avoid displacements, e) lamination and curing for adhesive activation and/or polymerization of prepreg layers. Another option for increasing process accuracy is represented by the use of laser drilling after lamination: the coverlay film is applied without pre-drilling; after lamination, laser drilling is performed in order to selectively eliminate the coverlay film and obtain the designed geometry [6]. However, with this approach, there are no advantages for the automation of the peeling phase.
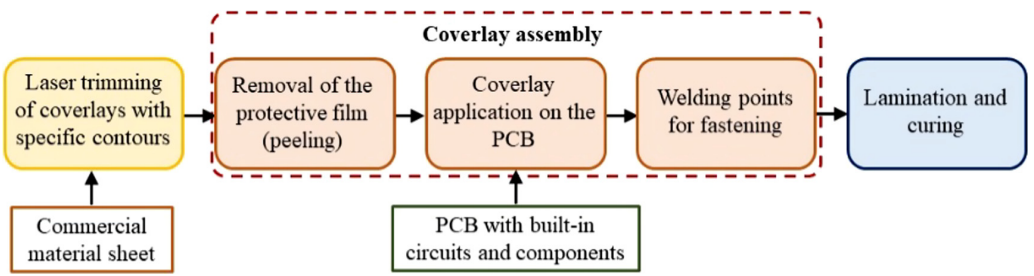

Fig. 5. Coverlay assembly: state-of-the-art process phases.

In panel-based processes of electronics industry, automating the peeling of flexible materials still represents an issue [7]. In this paper, a new concept is proposed for automated manipulation, positioning and assembly of coverlays in flexible and rigidflexible circuit manufacturing, addressing in detail the peeling issue. Section 2 presents the conceived assembly procedure including gripping, peeling of the protective film and assembly on the current layer of the stack-up. Section 3 illustrates the concept of the automated assembly system with its architecture and main equipment, including gripper architecture. Section 4 reports the experimental tests performed for the peeling step of a typical coverlay and finally, conclusions are presented in Sect. 5.

\section{The Proposed Assembly Approach}

In this Section, a strategy for coverlay automated assembly is proposed, identifying firstly the process requirements, then the gripping features and the peeling strategy and, finally, the process phases are described. 


\subsection{Requirements}

The requirements of the assembly procedure are determined based on the manual process currently carried out and the material properties.

Flexible Component Manipulation. Film coverlays are characterized by extremely low thickness. The system shall be able to manipulate these components, avoiding bending and ensuring flat positioning on FPCB surface.

Compliance to the Geometry. Depending on the specific FPCB design, coverlays are characterized by different dimensions and planar shapes, thus, the gripping strategy shall enable the compliance with a wide variety of geometries.

Removal of the Protection Film. In the assembly task, the protection film must be removed before coverlay application; therefore, a methodology for the peeling of such a film shall be implemented.

Thermal Bonding: Film coverlay shall be constrained to the FPCB by applying one or more bonding points, by locally activating the epoxy resin with a soldering tool.

Performance. Accuracy and repeatability of the assembly process shall be lower than the accuracy limit determined for flexible circuits with High Density Interconnect (HDI) $\operatorname{PCB}(100 \mu \mathrm{m})[6]$.

\subsection{Gripping}

In the manipulation of flat surfaces characterized by extremely low thickness combined with low stiffness materials, thus, deformable, the issue of preserving component configuration, avoiding undesirable bending, arises. This aspect can be even more relevant during the positioning phase, in which folds and air bubbles must be avoided. The use of a flat gripping surface as interface with the component represents a valid option to address these aspects.

Gripping strategy shall also comply with the requirement of the selective pick. Compliant grippers usually rely on the 3D shape of the object, in order to passively adapt to perform gripping, using different approaches and often implementing soft materials [8]; there are also commercial products available enabling the unconditional pick of objects with flat surfaces [9]. In the application dealt with in this paper, coverlays lie on a flat surface; due to the extremely low thickness, their 3D shape is not appropriate to be passively distinguished from the background by a compliant gripper. Thus, the necessity of a strategy for gripper "pre-shaping" arises.

Basing on these considerations, the gripper specifically designed for coverlay "pickpeel-place" is characterized by a flat interface surface and based on vacuum principle; a pattern of holes, representing themselves a matrix of suction cups, is realized in the flat surface. In order to avoid the uncontrolled pick of other patches lying on the same support, as well as the possible vacuum loss due to uncovered condition of one or more orifices, each suction cup is controlled individually, thanks to an upstream pneumatic valve module. With this approach, gripping pattern can be set depending on each specific coverlay contour, enabling the possibility of gripping different geometries. 


\subsection{Peeling Strategy}

Peel strength represents one of the most important characteristics of coverlays, as it is a measure of component integrity in determined loading conditions. DuPont ${ }^{\mathrm{TM}}$ Pyralux $^{\circledR}$ LF coverlay material, for example, based on a Kapton ${ }^{\circledR}$ polyimide film, exhibits a peel strength of $1.6 \mathrm{~kg} / \mathrm{cm}$ after curing, defined by the provider as per the applicable standard [10].

On the other hand, peeling represents also one phase of the assembly, as the coverlay is provided coupled with a release material; it is also the most demanding challenge to address in order to accomplish the whole task, due to the uncertainties in the peeling dynamics combined with the geometrical variability of the patches. Peeling dynamics has been widely investigated by the scientific community, and there are a number of patented machines, designed to be implemented in roll-to-roll production for different applications, performing tape peeling. In the majority of cases, peeling is triggered by the action of rolls [11] or insertion means acting as blades [12], combined with high bending angles of the release material. However, circuit board industry is mostly characterized by panel-based processing and coverlays may have very different shapes and dimensions. Therefore, the necessity of implementing a specific material removal method arises, studied for components characterized by planar dimensions in the order of few square centimeters.

In order to trigger the peeling of a film, adhering to another due to a substrate of a bonding agent, a mechanical action should be applied. With decreasing thickness, this task becomes more demanding, requiring precision and tools with appropriate edges. The detachment is achieved when the force, properly applied, is sufficient to trigger local fracture of the bonding agent. In the approach hereafter presented, the issue is addressed by an inverted perspective: instead of applying a detaching force when precise positioning occurs, the method relies on driving the film towards a source of mechanical action, working continuously at a high frequency; the approaching is stopped when detachment takes place due to the contact randomly occurred between the film and the source of the force.

In the practical implementation, a rotary tool with appropriate cutting profile can be suitable. In our experiments, a rotary sanding drum was used as a trigger for the peeling of the release material. As shown in Fig. 6, such a method, called hereafter "prepeeling", exploits abrasive surface roughness to collide with the release material surface and detach it from the coverlay, thanks to the impulsive force obtained by high grinding speed and applied by one or more abrasive particles to the film to remove. Relying also on friction contact, mutual positions can be calibrated in order to guarantee that this force affects only the protective film. A specific setup, further described in this paper, was used to validate this method.

In Fig. 6, the most important process parameters are indicated. The $a$ parameter is the unsupported overhanging portion of the coverlay (cantilever configuration) out of the clamp jaws, while $e$ is the distance, along the x-axis, of the coverlay tip from the grinding tool axis, $r$ is the radius of the sanding drum, rotating with angular speed $\omega$ and exerting a torque " $T$ ". Parameter $e$ is straightly related to the approach angle $\vartheta$ to the cylindrical grinding tool, since $e=r \cos (\vartheta)$. These parameters have been chosen due to their direct relation with the physics behind the peeling process. Indeed, $a$ is related to 
a)

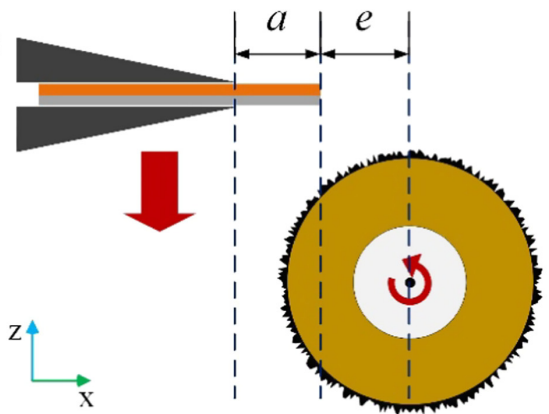

b)

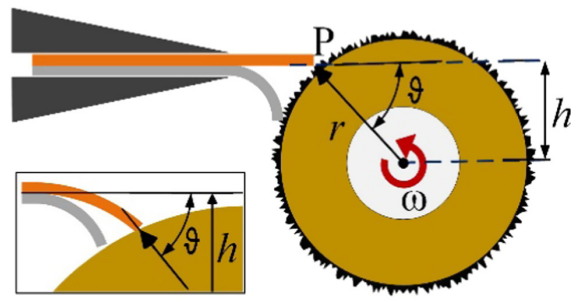

Fig. 6. Peeling trigger based on a sanding tool: a) the surface of the protective film approaches the rotating surface in a determined configuration; $b$ ) it is detached from the film coverlay. A magnification of the contact zone clarifies the definition of some parameters, considering the real operating conditions.

the coverlay flexural stiffness, so that stiffness decreases as $a$ increases. Parameter $e$ has influence on the peeling mechanism as it directly affects the direction of the interaction force applied by the tool on the component, due to the direct relation with angle $\vartheta$. The combination of $r$ and $T$ determines the value of the force and $\omega$ affects the momentum. Finally, the whole process is closely linked to the roughness of the sanding drum.

Parameter $h$ represents the distance, along the $\mathrm{z}$-axis, between the sample and the grinding tool. The optimal set $[a, \vartheta, h]$ uniquely identifies the most suitable configuration of the coverlay and the rotating sanding drum for the pre-peeling phase. Regarding this aspect, it is relevant to point out that, in the practical implementation, coverlay samples preserve the curvature of the original material reel. The real $h$ values differ from the theoretical value $h^{*}=r \sin (\vartheta)$ as they are affected by coverlay intrinsic curvature. According to this, $\vartheta$ is to be considered as the angle spanned between the radius connecting the center of the sanding drum with the contact point $\mathrm{P}$ and the axis of the clamp (or, in the automated task, of the end-effector), rather than the surface of the coverlay itself, as shown in the detail in Fig. 6-b).

\subsection{Assembly Procedure}

The assembly procedure is then defined in accordance with the chosen gripping and peeling strategy. With reference to Fig. 7, the following phases are identified:

a) Gripper Setup. With reference to the specific geometry of the coverlay to manipulate, an off-line setup is performed in order to activate selectively the vacuum orifices, thus modeling the gripping surface.

b) Coverlay Pick. Once the position of the component to manipulate is detected by an external sensing source, i.e. a vision system, the gripper can approach it and perform the pick on the coverlay side. A portion of the coverlay is kept on purpose outside of the gripping envelope.

c) Pre-Peeling. The unconstrained component portion is approached to the grinding tool, by the protective film side, thus triggering the detachment of the two films. 
a)

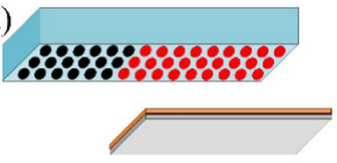

d)

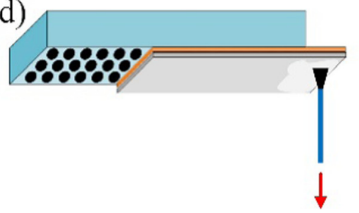

b)

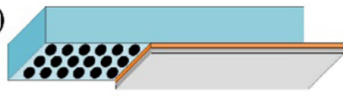

e)

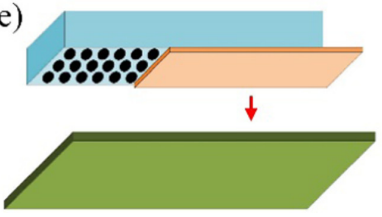

c)
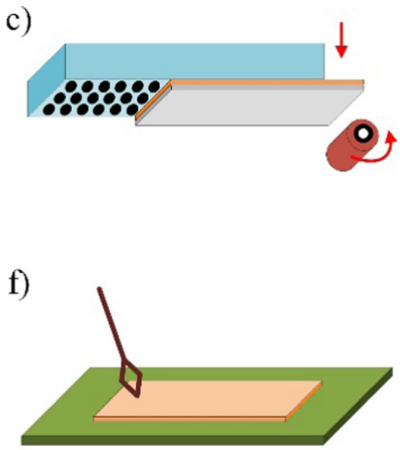

Fig. 7. The phases of the proposed assembly procedure: a) Gripper setup, b) Coverlay pick, c) Pre-peeling, d) Peeling, e) Coverlay positioning, f) Thermal bonding.

d) Peeling. The whole protective film is pulled away by a suction cup, acting on the component portion detached in the previous phase.

e) Coverlay Positioning. The coverlay is placed on the PCB.

f) Thermal Bonding. The coverlay is bonded in one or more points in order to guarantee constrained precise positioning before lamination.

\section{Assembly System Architecture}

The whole assembly system under study is based on the approaches proposed for gripping and pre-peeling. In this section, a concept of the workcell and the design of the specific gripper for the task are presented.

\subsection{Workcell Description}

The workcell architecture for coverlay application, outlined in Fig. 8, is aimed at exploiting the proposed automation strategy to obtain the PCB assembly ready for lamination and curing. The inputs of the process are the flex or rigid-flex PCB with circuits and SMT (surface mounted technology) components installed in previous steps.

In the assembly concept, the task is customized off-line, depending on the coverlay contour: a specific software tool is required for the pre-shaping phase. This setup is fundamental for task completion, since an appropriate portion of the picked coverlay has to remain outside of the gripper envelope, enabling peeling triggering.

In the workcell concept there are two conveyors, one providing the laser-trimmed coverlays and one sliding the flex or rigid-flex PCBs to assemble. The "pick-peel-place" task is performed by a commercial manipulator, equipped with the specifically designed gripper. The implementation of a collaborative manipulator is foreseen, due to the advantages provided by including a human operator in the assembly environment [13], even for a mere on-site supervision. Within the collaborative robot scenario, several commercial models are suitable for the task, considering the extremely low payloads and the accuracy required, assessed $100 \mu \mathrm{m}$ in Sect. 2.1. As an instance, in Figs. 8 and 9 the 
a)

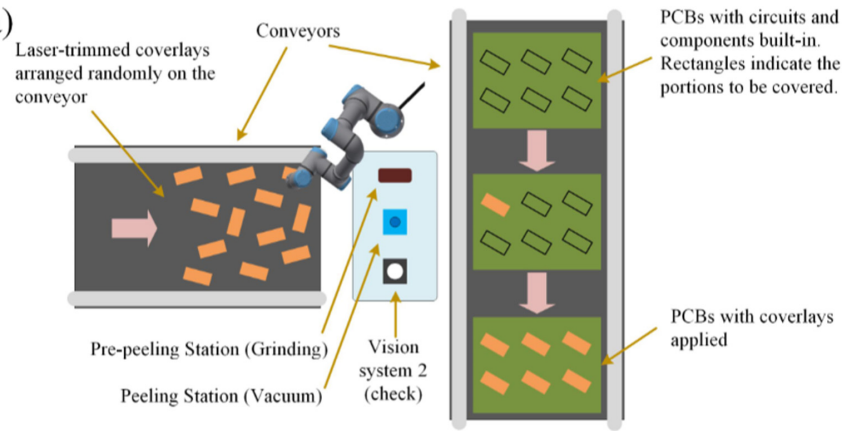

b)

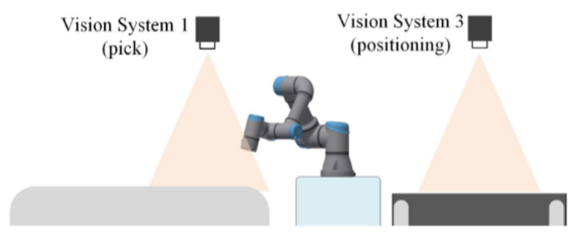

Fig. 8. Possible architecture of the workcell for coverlay application in a generic assembly scenario: a) top view and b) front view.

gripper is equipped on a Universal Robot UR5e. Regarding component pick and place, two vision systems are placed on top of the two conveyors, with appropriate fields of view (FOVs) to detect, respectively, position and orientation of the coverlay to pick, and the release position on the PCB. Within the robot reach, the pre-peeling and peeling stations are included, corresponding respectively to a rotating sanding drum and a fixed vacuum gripper; a vision system is necessary for gripping position check.

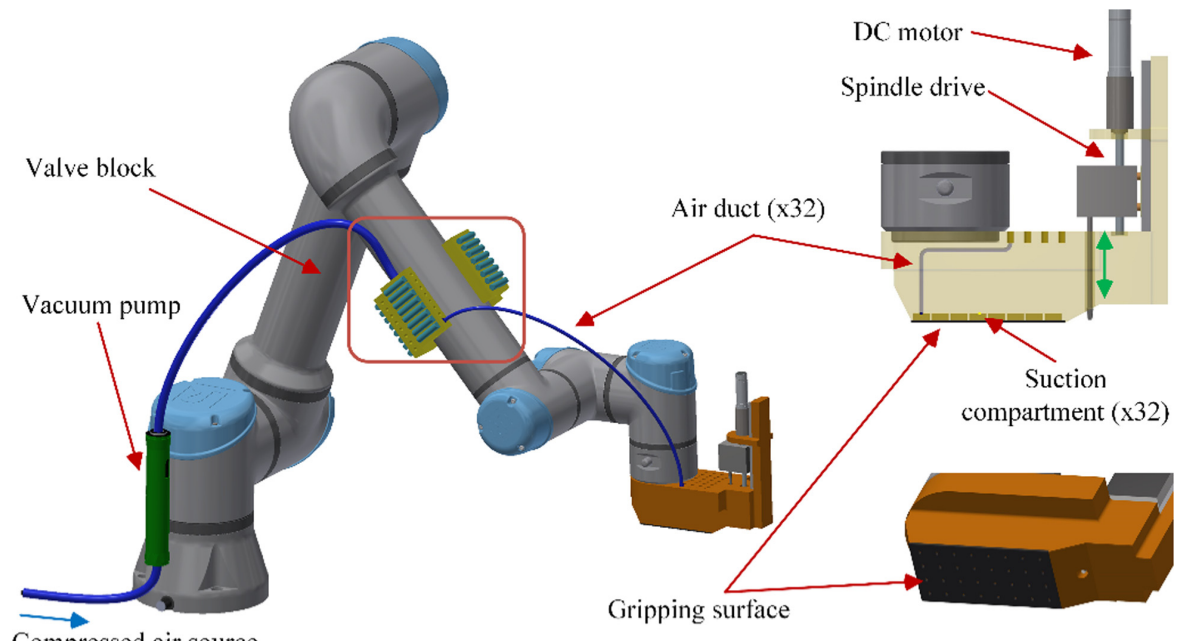

Compressed air source

Fig. 9. Design of the specific gripper. On the left, the gripper equipped on a UR5e; on top, right, gripper components are highlighted: on bottom, right, the interface surface is indicated. 


\subsection{Gripper Architecture}

A specific gripper is designed in order to perform coverlay film manipulation and comply with all the assembly phases. The overall design is shown in Fig. 9 and each subsystems is hereafter described.

Vacuum Pump. A vacuum pump, dedicated to gripper actuation, is installed on robot frame. In the design reported in Fig. 9, a vacuum generator is included.

Interface. The interface with the object consists of a flat surface; tiny holes provide vacuum source and a rubber coating guarantees adhesion and avoids undesired impacts with the board during the component placement. The diameter of the holes, $2 \mathrm{~mm}$, has been experimentally determined as a trade-off between gripping force and deformation of the material due to suction force.

Valve Block. The interface surface covers the suction compartments, which are independent each from another provided by the vacuum valve block. The valve block consists in a series of 32 tiny $3 / 2$ normally closed, cartridge solenoid valves, commercially available, supplied by common manifolds in parallel configuration, controlling, downstream, one duct each one; only one duct is highlighted in the Figure. Each duct ends up with a suction compartment. With this configuration, the goal of selectively shaping the gripping surface is achieved by modeling the gripping surface, controlling each valve individually.

Bonding Tool. The bonding apparatus consists in a retractile, metal solder tool. It can slide on the side of the gripper, in order to bond the coverlay once it is located on the $\mathrm{PCB}$, while it is maintained by the gripper in the correct position. One spindle drive actuated by a DC motor enables tool sliding, as shown in Fig. 9. The bonding tool slides out from a flat surface adjacent to the gripping surface, inclined to it in order to work as a foothold for the vacuum peeling tool during peeling completion.

\section{Peeling Tests}

\subsection{Experimental Setup}

As stated above, the peeling of the coverlay is conceived in two steps: pre-peeling and peeling completion. In order to verify the effectiveness of the proposed method to trigger coverlay peeling, a series of experimental tests was performed. The following parameters are considered to mainly affect the process: thickness of the coverlay, grit designation and dimensions of the sanding drum, rotational speed, geometrical configuration of the coverlay/drum approach. In this phase, the feasibility is addressed, focusing on the individuation of the optimal geometrical configuration for a defined set of coverlay material (thickness), pre-peeling tool (grit designation and diameter) and rotational speed.

Coverlay material is DuPont Pyralux LF0110 made of $25 \mu \mathrm{m}$ thick Kapton polyimide coated on one side with $25 \mu \mathrm{m}$ proprietary modified acrylic adhesive. The adhesive 
side is covered by a protective film with thickness of $40 \mu \mathrm{m}$. Coverlays are IPC certified by the supplier. Coverlay geometries are determined on the basis of an industrial case, currently object of study of the research group; such a geometry is rectangular with dimensions $25 \times 40 \mathrm{~mm}$; further materials and dimensions will be investigated in following experimental surveys. The samples are trimmed, which means full-thickness cut, by a 2D laser cut machine from a coverlay sheet with dimensions $610 \times 454 \mathrm{~mm}$, obtained by cutting a roll $610 \mathrm{~mm}$ wide and 76 meters long.

The laser trimming operation separates the coverlays from the sheet, then collected and loaded on a conveyor for the subsequent operation. The testing equipment is composed by two main parts: 1) apparatus for coverlay positioning; 2) pre-peeling stage. With regard to the first component, the coverlays samples are mounted on a three-axes high precision metric stage, actuated by three micrometer screws, with travel range of $15 \mathrm{~mm}$ on each axis and sensitivity $1 \mu \mathrm{m}$. The coverlay samples are held inside two small aluminum jaws mounted at the end of two cylindrical fixtures. In order to distribute the clamping force on a higher coverlay surface, two stainless steel plates with thickness $0.45 \mathrm{~mm}$ are used as interfaces between the clamp jaws and the coverlays. The pre-peeling stage consists of an electrical drilling tool, mounted horizontally. The sanding drum is a cylinder with an external diameter of $8.78 \mathrm{~mm}$ and height $12.7 \mathrm{~mm}$ ( $1 / 2$ in.) used for smoothing applications, with grit designation P80, corresponding to average particle diameter of $201 \mu \mathrm{m}$. The tool shaft has a diameter of $3.2 \mathrm{~mm}$. The setup is showed in Fig. 10.
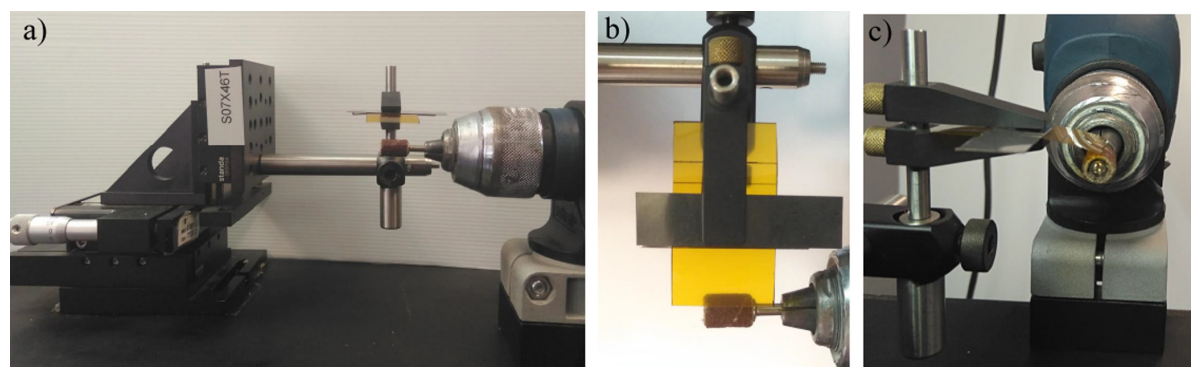

Fig. 10. Peeling initialization experimental set-up. Frontal (a), top (b), side view (c).

Preliminarily, the geometry of the set-up is verified: vertical stroke of the coverlay, alignments between grinding tool axis and coverlay profile, zeroing of the z-axis coincident to the first contact between the coverlay protective layer surface (bottom) and the side of the cylindrical grinding tool, centered position of the grinding tool with the coverlay width, etc.

The series of experimental tests was based on the variation of parameters $a$ and $e$; $\omega$ was set at $800 \mathrm{rpm}$ and the same sanding drum - thus, same radius and same sanding grade - was used. $a$ was set at 10 and $15 \mathrm{~mm}$, while $e$ was set at 0,1 , and $2 \mathrm{~mm}$; for $\mathrm{a}=10 \mathrm{~mm}$ also e $=1.5 \mathrm{~mm}$ was considered as a further set of variables. Each of the 7 parameter sets is tested with 5 repetitions (sample labels $\mathrm{A}, \ldots, \mathrm{E}$ ). This value is reputed sufficient for the feasibility assessment. Each run is performed by setting the parameters ( $a$ and $e$ ) and driving the coverlay sample towards the grinding tool by slow 
vertical motion (approximately $2 \mathrm{~mm} / \mathrm{min}$ ), as schematically shown in Fig. 6; vertical motion is stopped when peeling is triggered. The outputs of each experiment are the peeling triggering success, the peeled area and the recorded value of the parameter $h$; the standard deviation characterizing this value for each set of runs is indeed used to assess the repeatability of the process. For each successful pre-peeling test, an image was captured and processed in order to calculate the peeled area, considered an important parameter, since peeling completion relies on the gripping of a film portion already peeled.

\subsection{Results and Discussion}

Figure 11 shows the results of image processing procedure previously described, obtained for two runs. The light brown portion of images are pre-peeled areas (Fig. 11, left). They have irregular contours which are identified by image processing algorithms (Fig. 11, right). The proposed procedure can also be automated and used real-time for peeled area detection and characterization. Accuracy can be increased by illumination and background optimization in the image acquisition step.
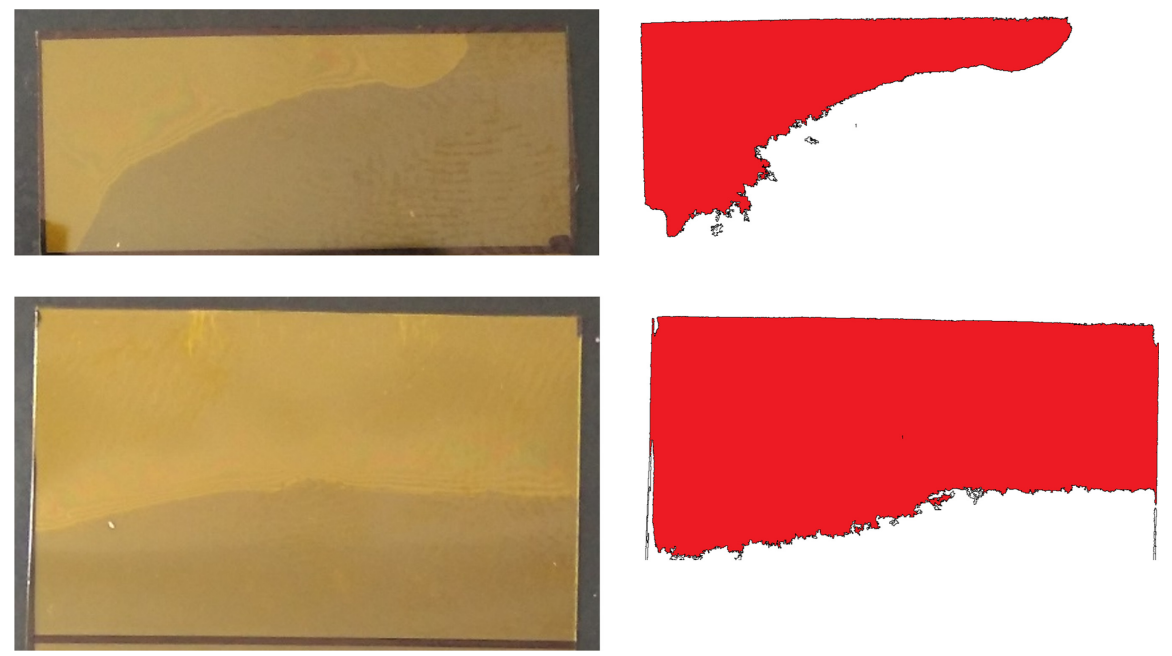

Fig. 11. Image processing applied to peeled samples. ROI (Region of Interest) original image (left) and processed image (right) for calculation of peeled area, in white. Runs reported: $100 \mathrm{~A}$ $(\mathrm{a}=10 ; \mathrm{e}=0$; sample $\mathrm{A})$ on top and 150C $(\mathrm{a}=15 ; \mathrm{e}=0$, sample $\mathrm{C})$ on bottom.

Table 1 reports the results obtained with the different parameter sets. At a first glance, it highlights that the grinding-based method can be a successful solution for peeling triggering in several configurations. It is important to point out that all the successful pre-peeling runs led to successful peeling completion, definitely validating the approach of splitting the peeling issue in two sub-phases.

Besides the validation of the approach, this preliminary series of tests was aimed at the definition of a reliable configuration for the automation of the pre-peeling phase, 
Table 1. Experimental results of peeling triggering.

\begin{tabular}{l|l|l|l|l|l|l|l}
\hline $\begin{array}{l}a \\
{[\mathrm{~mm}]}\end{array}$ & $\begin{array}{l}e \\
{[\mathrm{~mm}]}\end{array}$ & $\vartheta$ & Success Index & $\begin{array}{l}<h> \\
{[\mathrm{dm}]}\end{array}$ & $\begin{array}{l}\sigma_{h} \\
{[\mathrm{~mm}]}\end{array}$ & $\begin{array}{l}<\mathrm{PA}> \\
{\left[\mathrm{mm}^{2}\right]}\end{array}$ & $\begin{array}{l}<\mathrm{PA} / \mathrm{TA}> \\
\%\end{array}$ \\
\hline 10 & 0 & $90^{\circ}$ & $5 / 5$ & 5,54 & 0,17 & 129 & $52 \%$ \\
\hline 10 & 1,0 & $77^{\circ}$ & $5 / 5$ & 5,91 & 0,27 & 93 & $37 \%$ \\
\hline 10 & 1,5 & $70^{\circ}$ & $3 / 5$ & 6,02 & 0,17 & 171 & $68 \%$ \\
\hline 10 & 2 & $63^{\circ}$ & $3 / 5$ & 5,22 & 0,07 & 115 & $46 \%$ \\
\hline 15 & 0 & $90^{\circ}$ & $5 / 5$ & 6,79 & 0,22 & 174 & $46 \%$ \\
\hline 15 & 1 & $77^{\circ}$ & $2 / 5$ & 7,40 & 0,23 & 31 & $8 \%$ \\
\hline 15 & 2 & $63^{\circ}$ & $1 / 5$ & 6,40 & - & 89 & $24 \%$ \\
\hline
\end{tabular}

Success Index: number of successful pre-peeling runs; PA: Peeled Area; TA: Total Area (limited to the overhanging coverlay portion); $<h>$ : mean $h$ value and $\sigma_{\mathrm{h}}$ : standard deviation.

intended as the mutual position of an end-effector holding the coverlay and the grinding tool. The choice is the result of a hierarchical parameter analysis. The first parameter to consider is the Success Index: only configurations leading to $100 \%$ successful runs were considered. The Peeled Area PA (and, in particular, the ratio between PA and TA, the Total Area) was assessed as an important discriminating factor for the subsequent peeling completion phase; however, as already stated, all the successful pre-peeling runs were followed by successful peeling completion. From the pre-peeling automation perspective, acquired position standard deviation $\sigma_{h}$ is then relevant: since the $h$ value indicates a position suitable for the robot end-effector, a lower $\sigma_{h}$ is directly translated in a better repeatability of the process, that represents a fundamental indicator in an automated task. According to this approach, the most suitable set of parameters for the batch of coverlays currently under examination, is represented by the "100" series $(\mathrm{a}=$ $10 \mathrm{~mm} ; \vartheta=90^{\circ} ; \mathrm{h}=5.5 \mathrm{~mm}$ ). It is worth to point out that, according to the discussion reported in Sect. 2.3, in the practical implementation, this particular configuration $(\vartheta=$ $90^{\circ}$ ) does not correspond to the protective film surface tangential to the sanding drum, due to the coverlay intrinsic curvature. As a further remark, in all the successful runs, no damages were observed on coverlay inner surfaces.

In the proposed approach, the process is considered stochastic, relying on the sand particles random distribution. The coverlay/particles contact frequency is directly related to the high rotational speed of the tool. Certain contacts lead to detachment, thus, success probability is strongly increased by high rotational speeds. However, coverlay/tool relative configuration is a fundamental factor to enable the engagement of the only protective film layer; in some cases, indeed, also the coverlay film is engaged itself, leading to a process fail. The experimentation led to the optimal configuration for the specific case-study. 


\section{Conclusions}

The presented research activity deals with the automation of a specific task in electronic assembly, still performed manually. The topic is of outstanding interest, as it represents a critical phase in the production of flex and rigid-flex PCBs, whose implementation is incredibly growing in a wide range of application fields. Coverlays work as stiffeners, insulators and mechanical protection of the circuits; film coverlays present the most suitable mechanical properties, but they are characterized by low accuracy, as their application relies on operator skills. Furthermore, the removal of the protective film is troublesome and stressing.

The automation of the coverlay application phase is addressed by proposing an approach for the protective film peeling triggering, introducing a pre-peeling phase, based on a common workshop sanding tool. The whole assembly process is then outlined and the concept design of a suitable gripper is presented. Finally, the results of a series of tests, aimed at validating the peeling strategy and defining an optimal position for the automated pre-peeling phase, are reported.

The results demonstrate that the proposed peeling strategy can represent a successful methodology to be implemented in an automated process. Geometrical parameters need to be properly configured to ensure process reliability, defining the optimal relative position to promote the separation by engaging only the support material layer. In our experiments, indeed, some configurations led to $100 \%$ pre-peeling success, followed by successful peeling completion.

In future developments, the results obtained will be further validated in other experimental surveys; in particular, the variation of tool rotation speed will be considered, as well as the use of different sanding drums, corresponding to the variation of tool radius and sanding grade, and different coverlay materials. At the same time, gripper design will be refined, followed by the supply of all the components and realization. By equipping the gripper on a manipulator, it will be possible to validate the automation of most of process phases. Finally, the robot will be integrated in a complete workcell, as outlined in the paper.

Acknowledgments. This research was funded by the Italian Government through the National Operative Program H2020 PON 2014-2020, within the project "High-performance electronic Embedded Systems (HELMS)", grant number F/050507/02-03/X32.

\section{References}

1. Wong, W.S., Salleo, A.: Flexible Electronics: Materials and Applications. Electronic Materials: Science \& Technology. Springer, Boston (2009). https://doi.org/10.1007/978-0-387-743 63-9

2. Jillek, W., Yung, W.K.C.: Embedded components in printed circuit boards: a processing technology review. Int. J. Adv. Manuf. Technol. 25(3-4), 350-360 (2005)

3. Franke, J.: Three-Dimensional Molded Interconnect Devices (3D-MID): Materials, Manufacturing, Assembly and Applications for Injection Molded Circuit Carriers. Carl Hanser Verlag GmbH Co KG, Germany (2014) 
4. Canal Marquesa, A., Cabrera, J.M., de Fraga Malfatti, C.: Printed circuit boards: a review on the perspective of sustainability. J. Environ. Manage. 131, 298-306 (2013)

5. Khan, Y., et al.: Flexible hybrid electronics: direct interfacing of soft and hard electronics for wearable health monitoring. Adv. Funct. Mater. 26, 8764-8775 (2016)

6. Coombs, C.F., Holden, H.T.: Printed Circuits Handbook, 6th edn. McGraw-Hill, New York, USA (2009)

7. Plovie, B., et al.: Stretchable mold interconnect optimization: peeling automation and carrierless techniques. IEEE Trans. Compon. Packag. Manuf. Technol. 9(5), 955-962 (2019)

8. Shintake, J., Cacucciolo, V., Floreano, D., Shea, H.: Soft robotic grippers. Adv. Mater. 30(29), 1707035 (2018)

9. Joulin Vacuum Handling. https://www.joulin.com/all-industries/standard/minigrip.html. Accessed on 30 Sep 2019

10. IPC-TM-650 Test Methods Manual, 2.4.9 - Peel Strength, Flexible Dielectric Materials. IPC Association Connecting Electronics Industries (2014)

11. Toyoda, Y., Nobuyuki, T. (NGK Spark Plug Co., Ltd.). United States Patent: Method and Apparatus for Producing a Wiring Board including Film Peeling. US 7,481,901 B2 (2009)

12. Ahn, K.H. (Samsung Display Co., Ltd.). United States Patent: Method and Apparatus for Peeling Donor Film from Substrate. US 8,877,006 B2 (2014)

13. Ruggeri, S., Fontana, G., Basile, V., Valori, M., Fassi, I.: Micro-robotic handling solutions for PCB (re-)manufacturing. Proc. Manuf. 1, 441-448 (2017)

Open Access This chapter is licensed under the terms of the Creative Commons Attribution 4.0 International License (http://creativecommons.org/licenses/by/4.0/), which permits use, sharing, adaptation, distribution and reproduction in any medium or format, as long as you give appropriate credit to the original author(s) and the source, provide a link to the Creative Commons license and indicate if changes were made.

The images or other third party material in this chapter are included in the chapter's Creative Commons license, unless indicated otherwise in a credit line to the material. If material is not included in the chapter's Creative Commons license and your intended use is not permitted by statutory regulation or exceeds the permitted use, you will need to obtain permission directly from the copyright holder.

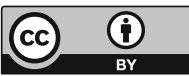

